\title{
FORUM
}

\section{Einige Bemerkungen zu Ideographie und Alphabetschrift}

\author{
Christian Stetter
}

Peter Schlobinski hat in einem kürzlich in der ZS erschienenen Beitrag (Schlobinski 2001) eine Deutung der chinesischen Schrift vorgetragen, in der er für eine Sichtweise des Funktionsprinzips dieser Schrift plädiert, die mit der herkömmlichen Auffassung bricht, es handle sich hier um eine Ideographie. In diesem Beitrag setzt er sich auch mit der Deutung der chinesischen Schrift auseinander, die ich in meinem Buch ,Schrift und Sprache" in einer Reihe von Bemerkungen skizziert habe. Thema von Schrift und Sprache war allerdings nicht das Chinesische oder Japanische, sondern die Alphabetschrift. Und die Problemstellung des Buchs war keine sprach- oder schrifttheoretische, sondern eine philosophische: Was, ist' die Alphabetschrift im Sinne des sokratischen $t i$ estin? In welcher spezifischen Weise hat dieser Schrifttyp unser Denken geprägt, namentlich unsere Auffassung von Grammatik und Sprachwissenschaft. Die gelegentlichen Seitenblicke auf die Schriftsysteme des Chinesischen und Japanischen haben lediglich dem Zweck gedient, eine Perspektive auf den Gegenstand meiner Überlegungen zu gewinnen, die selbst nicht schon ,,alphabetzentriert" angelegt war. ${ }^{1}$

Es ist für einen Autor immer erfreulich, wenn seine Thesen diskutiert werden, selbst wenn der, der sie diskutiert, zu einem anderen Resultat kommt wie hier. Namentlich setzt sich Schlobinski mit zwei Thesen meines Buchs auseinander, einmal der von mir vertretenen traditionellen ${ }^{2}$ Auffassung, die chinesische

$1 \mathrm{Zu}$ einer tiefergehenden Auseinandersetzung mit dem Chinesischen oder Japanischen fehlen mir die einschlägigen sinologischen bzw. japanologischen Kenntnisse, über die man in diesem Fall verfügen müsste. Immerhin habe ich mir für meine Ausflüge in dieses fremde Gebiet japanologischen Rat geholt und, soweit dies möglich war, einschlägige Literatur zu Rate gezogen.

2 Vgl. z. B. die folgenden Lexikoneinträge: ,Ideogramm: Schriftzeichen, das nicht eine bestimmte Lautung, sondern einen ganzen Begriff repräsentiert (z. B. bei Hieroglyphen oder der chinesischen Schrift).“ „Ideographie: Schrift, deren Elemente Ideogramme sind. Ideograph. Schriften können von Völkern verschiedener Sprache benutzt werden, da in den Schriftzeichen beim Lesen Begriffe erkannt werden und nicht Lautwerte." (Meyers großes Taschenlexikon in 24 Bänden 1987: 163). 
Schrift sei eine Ideographie - eine Auffassung, die sich explizit schon bei W.v. Humboldt findet -, zweitens und vor allem mit der von mir in der Tat geäußerten Ansicht, im Kulturkreis der chinesischen Schrift habe sich weder eine formale Logik noch eine Grammatik in ,,unserem“ westlichen Sinn ausgebildet. Diese These wird bei Schlobinski allerdings zur ,Annahme einer mangelnden Logikund Grammatiktradition", was - wie sich zeigen wird - nicht dasselbe ist.

Schlobinskis Kritik meiner Auffassungen scheint mir nun nicht so sehr auf Unterschieden in der Auffassung des empirischen Materials zu beruhen wie auf grundsätzlichen kategorialen Missverständnissen. ${ }^{3}$ Daher möchte ich diese im Folgenden aufgreifen und, soweit dies hier möglich ist, klären. Die hierbei unvermeidliche Auseinandersetzung mit Schlobinskis Lektüre meines Textes habe ich, um die Diskussion der beiden eben genannten Punkte nicht durch sachfremde Erörterungen zu unterbrechen, soweit es ging, in die Anmerkungen verlagert.

\section{Ist die chinesische Schrift eine Ideographie?}

Im klassischen Verständnis ist eine Ideographie eine ,Begriffsschrift', so schon bei W. von Humboldt. ${ }^{4}$ Diese Bezeichnung orientiert sich an der klassischen Sprachauffassung, für die das Nomen das Paradigma des Wortes war und die das, was das Nomen ,bedeutet", als Begriff auffasste. ${ }^{5}$ Das Wort „Haus“ bedeutete oder bezeichnete somit den Begriff des Hauses, das Wort ,Schrift“ den

3 Diese scheinen mir zumindest zum Teil auch auf selektiver Lektüre zu beruhen. Wie man das, was ich als ,linguistisches Relativitätsprinzip" beschrieben habe - es hängt vom Formenreichtum und der kategorialen Anlage eines Schriftsystems ab, was es als sprachliche Tatsache zu erfassen vermag (Stetter 1999: $131 \mathrm{ff}$.) -, in der Tradition des whorfschen Relativitätsprinzips oder gar der Positionen Havelocks oder Logans zu verorten vermag, das verstehe ich nicht. Dieses Prinzip gilt für jedes beliebige Schriftsystem, das ein Linguist zur Repräsentation sprachlicher Sachverhalte benutzt. Wie daraus ,whorfsche' Konsequenzen folgen sollen, sehe ich nicht. Aus ,So war die Entwicklung“" folgt doch nicht „So musste sie sein und anders ging es nicht.“ Vgl. dazu auch unten. I. ü. habe ich in den beiden letzten Abschnitten des Humboldt-Kapitels das Problem des Eurozentrismus in dessen Philosophie der Grammatik explizit angesprochen (Stetter 1999: 501 ff.).

4 Vgl. W.v. Humboldt (1969: 87ff. und 1906: $300 \mathrm{ff}$ ).

5 onoma ist bei Aristoteles terminus technicus für jede Art von Wort, das als logisches Prädikat verwendet werden kann. Daher in der Poetik (1457b) das kyrion onoma, was einfach „das normal verwendete Wort“ (im Gegensatz zum metaphorisch verwendeten) bedeutet. 
Begriff der Schrift usw. ${ }^{6}$ Eine Ideographie im allgemeinen Sinne ist somit eine Schrift, die mittels bestimmter Zeichen bestimmte Begriffe darstellt. In diesem Sinne benutzt man die Zeichen ,1“, ,2“, ,3“ usw. in der Mathematik als Unterscheidungen von Anzahlen oder Maßen, also als Begriffe. Statt ,die Kardinalzahl zwei“ können wir auch schreiben ,die Kardinalzahl 2“. Der Unterschied beider Schreibweisen zeigt sich, wenn wir die Sprache wechseln: „,the cardinal number two": „the cardinal number 2“. Im Gegensatz zu den Zeichen ,zwei“ oder „two" bezeichnet das Zeichen „2" kein Wort einer bestimmten Sprache, sondern das betreffende Objekt des Bezugnahmegebietes direkt. Die Ideographie ist also - im Gegensatz zur Bilderschrift oder zur Piktographie - durch eine $1: 1$-Beziehung von Zeichen und Bedeutung charakterisiert. Die Bedeutung , $y^{*}$,existiert" nur in dem Zeichen y. ${ }^{7}$ Diese Eigenschaft teilt die Ideographie mit der Alphabetschrift. Wird nun ein empirisch gegebenes, historisch gewachsenes Schriftsystem wie das Chinesische als Ideographie aufgefasst, so sind mehrere Zusatzbedingungen zu beachten: Erstens geschieht auch hier die Repräsentation der Bedeutung stets mittels konventionalisierter Zeichen. Selbst die einfachsten, in der Literatur gelegentlich als Piktogramme oder Ideogramme ${ }^{8}$ bezeichneten Zeichen wie die für ,Mensch", ,,eins", ,,zwei“"

6 Frege hat diese Auffassung in zwei Punkten modifiziert: Erstens gehört zum Begriff, dass er - wie der Funktionsterm - eine syntaktische Leerstelle hat, in die ein Argument eintreten kann, auf das der Begriff angewendet wird. Also ,sieht" der Begriff etwa so , aus“": ( ) ist P. Zweitens kann Begriff jeder sprachliche Ausdruck sein, der, ein Name für ein bestimmtes Argument gegeben, z. B. der Name ,Zeitschrift für Sprachwissenschaft“",

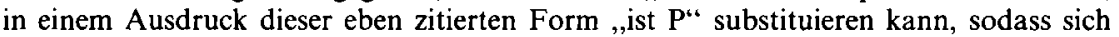
zusammen mit dem (quantifizierten) Argumentnamen ein wahres oder falsches Konstativum ergibt, z. B. „Die Zeitschrift für Sprachwissenschaft wird von der Deutschen Gesellschaft für Sprachwissenschaft herausgegeben." Auch dieser hier kursiv gedruckte Ausdruck ist in logischer Hinsicht ein, allerdings komplexer, Begriff. Insofern hat sich mit dieser Revision auch der ganze Psychologismus erledigt, der mit der älteren Auffassung des Begriffs Begriff verbunden war. Es geht nur um sprachliche Ausdrücke und deren Verwendung in logisch normierten Zusammenhängen.

7 Das Auftreten von Homonymie tangiert dieses Prinzip nicht.

8 Was Schlobinski unter ,Ideogramm" versteht, vermag ich seinem Text nicht genau zu entnehmen. Ich habe jedenfalls, wo ich vom Chinesischen oder von den Kanji gesprochen habe, unter einem Ideogramm kein Element einer Bilderschrift oder einer Piktographie verstanden, sondern davon gesprochen, dass es sich hierbei um konventionalisierte Formen handelt, von denen manche ,sich auf bildliche Darstellungen“ von Dingen oder Lebensbereichen ,zurückführen lassen“ (Stetter 1999: 50). Ich sehe nicht, wie diese Position im geringsten abwiche von der, die Schlobinski am gleichen Ort vertritt, z. B. S. 127: „Die rechte Komponente [des Zeichens für „Wein“, Ch. St.] stellt ursprünglich einen (Wein)Krug dar."

Möglicherweise orientiert sich Schlobinski in seinem Sprachgebrauch an in der Literatur begegnenden Übersetzungen der chinesischen Einteilung der Schriftzeichen in xiàng xíng ("ursprüngliche Bilder", gelegentlich übers. mit „Piktogramm", z. B. rén, Mensch, oder ri, Sonne), zhĭ shi (,,symbolische Bilder", gelegentlich übers. mit ,Indikator"“, z. B. sān, drei), huì yi (,,zusammengesetzte Bilder"), xíng shēng (Phonogramme) usw. (vgl. Müller-Yokota 1994: 362 ff.). Diese Klasseneinteilung ist jedoch rein historisch, sie 
usw. sind vollständig konventionalisierte Zeichen, deren semantischer Wert sich aus ihrer Stellung im System ergibt. ${ }^{9}$ Zweitens repräsentieren diese Zeichen nicht nur Begriffe, sondern semantische Werte, und zwar jedes Han ze bzw. Sinographem oder Kanji genau einen. Dies wird eindrucksvoll durch die Tatsache bestätigt, dass es bei vielen Kanji verschiedene „Lesarten“ gibt. Der semantische Wert des Schriftzeichens besteht genau darin, dass es diese verschiedenen Lesarten zulässt und damit semantische Alliterationen wie etwa beim Namen der nordjapanischen Stadt Sendai, die in der alphabetischen Transkription verlorengehen.

Drittens repräsentiert - damit kommen wir zum ersten Kernpunkt der Kontroverse - das Chinesische zwar Morpheme bzw. Wörter. Ein Sinographem stellt ein Morphem bzw. Wort dar in dem folgenden Sinne: Es ist ein Wort bzw. Morphem (nämlich der Schriftsprache). Es stellt aber nicht die phonematische Artikulation des entsprechenden Wortes der oralen Sprache dar. ${ }^{10}$ Dies macht die wesentliche Differenz aus, die jede Ideographie von der Alphabetschrift und verwandten Schriftsystemen unterscheidet. Die Alphabetschrift stellt die Bedeutung des - wohlgemerkt schriftlichen - Wortes dar, indem sie uno actu ein graphisches Analogon der phonematischen Artikulation der korrespondierenden Einheit des oralen Sprachsystems gibt. Damit organisiert dieser Schrifttyp eine Rückkopplung von schriftlicher und oraler Sprache besonderer Art. Sie schränkt, genauer gesagt, die Extension der der Schriftsprache zuzuordnenden oralen Dialekte mehr oder weniger stark ein oder erzwingt, genauer gesagt, die Ausbildung eines vom Schriftlichen her konstruierten Standarddialekts. Ein Phänomen wie die Koexistenz von On- und Kun-Lesarten im Japanischen, wo ein und demselben Zeichen Wörter verschiedener Etymologie zugeordnet werden, einmal ein Wort chinesischen Ursprungs (On-Lesart), zum anderen japanischen Ursprungs (Kun-Lesart), ${ }^{11}$ wäre in der Alphabetschrift ebenso undenkbar wie die Tatsache, dass sich in China Millionen von Menschen, die die

sagt nichts aus über die Funktionsweise der betreffenden Zeichen im System. Das Sinogramm für rén stellt ebenso den semantischen Wert des Wortes, ,rén“ "bzw. den Begriff ,rén' (Mensch) dar wie das für rì (Sonne) den Begriff ,Sonne' darstellt. Dasselbe gilt für jedes Phonogramm. In diesem Sinne handelt es sich bei all diesen Zeichenkategorien um ,Ideogramme' in dem von mir so auch verwendeten Sprachgebrauch (vgl. Stetter 1999: 132f. und 456).

$9 \mathrm{Vgl}$. Stetter (1999: 472).

10 Vgl. Stetter (1999: 132f.). Schlobinski behauptet, die Auffassung, das Chinesische sei eine ideographische Schrift, beruhe ,,in einer postulierten [...] Semantizität der Signifika" (S. 129), d.h. derjenigen Komponente eines sogenannten Phonogramms, das einen Hinweis auf den semantischen Wert gibt. Ich sehe nicht, wo ich diese These vertreten hätte (sie macht auch keinen Sinn). Der Unterschied ist rein negativer Art: Eine Ideographie repräsentiert nicht die phonematische Artikulation der dem schriftlichen Zeichen im oralen System korrespondierenden Einheit (Morphem, Wort).

11 Z. B. ,Berg, Gebirge: san (On), yama (Kun). 
verschiedensten Dialekte sprechen - mit europäischen Kategorien hätten wir diese als verschiedene Sprachen wie Deutsch, Englisch, Niederländisch usw. anzusprechen - mit und in einem einzigen Schriftsystem kommunizieren können.

Seine Kritik an dem vorgeblichen ,ideographischen Mythos" in der Auffassung der chinesischen Schrift (dem auch ich aufgesessen sei) stützt Schlobinski auf eine Interpretation der im Chinesischen wohl häufigsten Zeichenkategorie, des in ,,westlicher“ Terminologie sogenannten Phonogramms ${ }^{12}$ oder Determinativphonetikums. Es besteht aus zwei Elementen, die teilweise - soweit es sich nicht um Radikale handelt - auch allein als vollgültige Zeichen verwendet werden können. ${ }^{13}$ Das eine von ihnen, Schlobinski nennt es "Signifikum“ (S. 128), hat die Funktion, die Bedeutung bzw. den semantischen Wert des Gesamtzeichens anzudeuten - wie auch immer, in der Regel durch Analogien auf der Ebene des Graphismus oder auch qua Konvention -, das andere Element, das „Phonetikum“, ist nach Schlobinski ,die Komponente eines Sinographems, die Hinweise auf seine Aussprache gibt, wobei unterschiedliche Grade von Phonetizität anzusetzen sind" (S. 128). Die entscheidende Frage lautet, wie es diese Hinweise gibt. Davon hängt $a b$, inwiefern und ob überhaupt hier von Phonetisierung die Rede sein kann.

12 Gelegentlich auch Logophonogramm genannt.

13 Vgl. hierzu das Beispiel bei Schlobinski S. 127f. Was Schlobinski über Radikale äußert (und über das, was ich darüber geäußert haben soll), ist mir in mehr als einer Hinsicht nicht nachvollziehbar. Zunächst: Die These, nach der , in Zeichen mit einem phonetischen und semantischen Anteil die semantische Komponente immer auch das Radikal darstelle" (S. 129), habe ich nirgends vertreten (vgl. allerdings dazu MüllerYokota 1994: 365). Schlobinskis Anmerkung 12 (S. 128), die sich wohl auf S. 133 meines Buchs bezieht, ist schlicht irreführend. Die Funktionsweise eines Phonogramms habe ich vielmehr anhand zweier Logogramme verdeutlicht (Stetter 1999: 133f.). Sodann: Selbstverständlich werden Radikale in Wörterbüchern dazu verwendet, ein Ordnungssystem für die Anordnung der Han ze bzw. Kanji zu konstruieren. Aber damit sie vom Lexikographen schließlich so verwendet werden konnten, mussten sie doch wohl unabhängig davon schon im Schriftsystem ausgebildet sein (vgl. etwa Müller-Yokota 1994: 379). Und in elaborierteren Wörterbüchern (vgl. z. B. New Japanese-English Character Dictionary) werden sie auch als eigene Schriftelemente aufgeführt, denen man zwar kein Wort der betreffenden oralen Sprache zuordnen kann, die aber nichtsdestoweniger als konventionalisierte Schriftelemente mit eigenem Wert im System der geschriebenen Sprache existieren. Dieser Wert kann nur umschrieben werden, er ist aber natürlich durch den Gebrauch ebenso exakt fixiert wie die eines ins Orale ,übersetzbaren“ Zeichens. In dieser Funktion, als die kleinsten im System der Han ze bzw. Kanji ausmachbaren Elemente mit Zeichenfunktion (Striche haben für sich ebensowenig Zeichenfunktion wie Buchstaben), habe ich das semantische Radikal als ein Schriftelement einer Ideographie erwähnt, für das es in der Alphabetschrift kein Analogon gibt und das in der chinesischen oder japanischen Lyrik auch in Weisen genutzt wird, die wir in unserer nicht kennen. Die relative Autonomie der Schrift gegenüber der korrespondierenden gesprochenen Sprache ist das Problem, dem ich in Schrift und Sprache S. $133 \mathrm{ff}$. nachgegangen bin. Was Herr Schlobinski da heraus- oder hineingelesen hat, bleibt sein Geheimnis. 
Einen Hinweis auf die ,Aussprache“ des Gesamtzeichens kann das Phonetikum nur aufgrund von Eigenschaften haben, die es nicht mit dem Signifikum teilt. Die Homonymie kann also in dieser Hinsicht keine Rolle spielen, obwohl sie die Grundlage des ganzen Geschäfts ist. ${ }^{14}$ Sie müssen also in den Besonderheiten der Zeichengestalt des Phonetikums liegen. Für jedes Signifikum kommen nur ganz bestimmte Zeichen als Phonetika in Frage, nämlich diejenigen, die kontingenterweise durch ihre ,Aussprache“ einen Hinweis auf die „Aussprache“ des Gesamtzeichens nahelegen. Was besagt nun der Begriff der Aussprache? Für alphabetzentrierte Ohren bzw. Denkorgane eine Selbstverständlichkeit: Wenn ich das Wort ,in“ lese, sagt mir die Schreibweise, wie ich es auszusprechen habe (das habe ich im Schriftunterricht der Grundschule gelernt): $/ \mathrm{i} / \mathrm{h} / \mathrm{n} /$.

Nun ist das Phonetikum aber nicht als Buchstabenfolge geschrieben, sondern als Komplex von Strichen, die nicht linear angeordnet sind, ${ }^{15}$ sondern deren Anordnung - den betreffenden Schreibkonventionen gemäß - bezogen ist auf einen imaginären Mittelpunkt der Gesamtfigur des Zeichens. Dieser Graphismus ist als Bild einer linearen Folge von Elementen wie etwa eine Folge von Artikulationsbewegungen oder von Phonemen oder Silben usw. von vornherein ungeeignet. Er ist nicht dafür gemacht, sondern dafür, eine Bedeutung zu repräsentieren, den semantischen Wert eines Wortes. Dieses leistet er durch eine besondere Konfiguration von Strichen. Sie unterscheidet diese Zeichenfigur von allen anderen desselben Schriftsystems. Kenne ich die Bedeutung dieses Zeichens und beherrsche ich zugleich im Mündlichen einen chinesischen oder japanischen Dialekt, so kann ich sagen: ,,Dieses Zeichen ... bedeutet (z. B.) „,shan“ oder „,yama“. Nun ist aber keiner der drei Striche, die (in diesem Beispiel) das Zeichen bilden, deren Konfiguration ich also die „Aussprachen“" „,shan" oder ,yama“" zuordne, durch eine Schreibregel irgendeinem Lautsegment der oralen Zeichengestalten ,,shan“ oder ,yama“ zugeordnet. Dieselben Striche werden in beliebig vielen anderen Zeichen mit völlig anderer ,,Aussprache" verwendet. Ja, dasselbe Zeichen kann - das Beispiel zeigt es - so oder so ,ausgesprochen" werden. Was ich lediglich weiß, ist, dass das gesamte Zeichen im Chinesischen durch das orale Wort ,shan", im Japanischen, so die Kun-Lesart angesagt ist, durch das orale Wort ,yama“ wiedergegeben oder gelesen wird. Und dass es so und nicht anders gelesen wird, weiß ich, weil ich bzw. insofern ich die Bedeutung des schriftlichen Zeichens ... und die ,korrespondierende" Bedeutung des oralen Zeichens ,shan“ bzw. „yama“ kenne und schriftliches und orales Zeichen (signe) qua Einheiten, beide

14 Das wird manchem paradox erscheinen, ist es aber nicht: Die Homonymie ist kontingent. Der Grund für das Funktionieren des Phonogramms muss aber ein systematischer sein.

15 Sie sind zwar in linearer Ordnung geschrieben, aber ihre Anordnung ist nicht linear. 
konstituiert durch die Abbildung eines bestimmten signifiant auf einen bestimmten signifié, ${ }^{16}$ einander zuordnen kann. Bei dem „Aussprache“ (eines Wortes) genannten Vorgang handelt es sich also tatsächlich um die Zuordnung eines Wortes der geschriebenen Sprache zu einem Wort der gesprochenen Sprache, um eine Übersetzung aus einem Medium ins andere. ${ }^{17}$

Unter Phonematisierung bzw. Phonetisierung versteht man aber in der Schrifttheorie etwas anderes: die Darstellung des signifiant eines gesprochenen Wortes durch konventionalisierte graphische Zeichen, die für sich als Namen für Lautfragmente gelesen werden können ${ }^{18}-$ auch wenn sie im Schriftgebrauch ganz anders verwendet werden, nämlich als distinktive Unterscheidungsmerkmale von graphischen Wortgestalten. Der Begriff des Buchstaben umfasst also zwei verschiedene Funktionen: erstens seine Funktion als Graphem, als (in der Regel) kleinste bedeutungsdifferenzierende Einheit eines Wortes der Schriftsprache; dies ist seine primäre Funktion. Zweitens seine Verwendung als Name für Laute bzw. Phoneme in speziellen Sprachspielen des Anfangsschriftunterrichts oder der Phonologie. Dies ist eine sekundäre Funktion, die aber häufig für die primäre gehalten wird. In dem oben erwähnten Beispiel ,in“ werden in der sekundären Funktion das $\langle i\rangle$ und das $\langle\mathbf{n}\rangle$ je für sich als Name für ein Phonem gelesen. Was Phonematisierung heißt, kann man - um im Bereich der chinesischen Schrift zu bleiben - an der Entwicklung der Kana aus aus dem Chinesischen übernommenen Zeichen sehen, wo der Schritt von der kontingenten Zuordnung eines oralen Morphems, d. h. Zeichens, zu einem geschriebenen Zeichen hin zur systematischen Verwendung einer abgewandelten Form dieses Zeichens als Namen für eine Silbe, d.h. einen signifiant, vollzogen wird. ${ }^{19}$ Und der koreanischen Buchstabenschrift kann man die Herkunft dieser gegenüber den Kana weitergehenden Phonematisierung aus einer Silbenschrift bis heute an der Gruppierung der Hangul-Zeichen ablesen.

Der Begriff des Phonetikums ist also, milde gesprochen, ein alphabetzentristisches Missverständnis. Er verstellt das Verständnis des im sogenannten Phonogramm Geleisteten eher, als dass er es erhellt. Von phonematischem Prinzip kann jedenfalls keine Rede sein. Wer das Phonetikum nicht als genuines Zeichen kennt oder nicht weiß, dass es hier in diesem komplexen Zeichen als Phonetikum

16 „Abbildung“ hier im loischen Sinne einer eindeutigen Zuordnung von Elementen einer Definitionsmente zu Elementen einer Wertemenge verstanden. Das schriftliche Zeichen muss also unabhängig von der signifié-Funktion des „korrespondierenden" oralen Wortes sozusagen „seinem“" signifié zugeordnet werden können, damit die Korrespondenz-Relation überhaupt etabliert werden kann.

17 Auch Müller-Yokota (1994: 364) verwendet diesen irreführenden Begriff, der wohl Tradition ist, kommentiert ihn aber doch als ,Lesung“, und das ist logisch etwas ganz anderes.

18 Das A, das Be usw.

19 Z. B. Kanji to (= Halt, halten) $\Rightarrow$ Silbenzeichen (Hiragana bzw. Katakana) ,to“. 
verwendet wird, dem hilft es beim Finden der ,Aussprache“ nicht das Geringste. Gelernt werden von den Anfängern ohnehin die ganzen Zeichenkomplexe als semantische Einheiten. Somit können die Phonogramme bzw. Determinativphonetika nicht als Argument dienen, auch die chinesische Schrift auf den bewährten Evolutionsgang der Phonematisierung zu befördern, und es muss wohl dabei bleiben, diese Schrift - mit den oben genannten Präzisierungen - als Ideographie und damit als Gegentyp zum Alphabet zu begreifen. ${ }^{20}$

\section{Chinesische Schrift und formale Logik}

Damit komme ich zum zweiten Punkt der Kontroverse, der Frage der Logik. Schlobinski zitiert meine diesbezügliche These einmal korrekt, nämlich dass sich im Kulturkreis der chinesischen Schrift keine formale Logik (und Grammatik) im, westlichen' Sinn entwickelt habe, einmal inkorrekt, in China hätte sich keine Logik entwickelt. ${ }^{21}$ Letztere widerlegt er. Meine These lautete jedoch - man möge nachlesen -, dass sich im Fernen Osten keine formale Logik entwickelt habe. ${ }^{22}$ Die Frage ist also, was unter einer ,formalen“ Logik zu verstehen ist.

20 Man sieht an diesem Beispiel, wie fatal es ist, wenn man sich ohne ein klares zeichentheoretisches Fundament, eine Symboltheorie in Goodmans Sinne etwa, an die Analyse derartiger Phänomene macht. Wie fremd Herrn Schlobinski solche Gedankengänge sind, zeigt sein Ausfall - anders ist das nicht zu nennen - gegen Roland Barthes' Empire des signes. Das Buch gerät ihm zu ,allerlei Phantasien und Spekulationen“ über Fernost, zu einer „Mythologisierung und Mystifizierung“ eines Reichs von Zeichen (S. 117). Er versteht nicht, was Barthes in diesem Buch geleistet hat: nämlich eine Phänomenologie der - mit Goodman zu sprechen - Bezugnahmehandlungen oder -akte, die Symbolschemata allererst zu Symbolsystemen und damit zu dem machen, was man in saussurescher Tradition ,signe“" nennt. Dabei ist dieser Gedanke doch in dem einleitenden Kapitel „Dort" deutlich ausgesprochen. Man muss allerdings die Sprache verstehen, in der dieses hellsichtige Buch geschrieben ist.

21 Ein analoger Lesefehler unterläuft Herrn Schlobinski auch auf S. 122f. seines Textes, wo er mir die Ansicht unterschiebt, dass ,das Chinesische keine Morphologie kenne“. Die Fußnote, auf die er sich dabei bezieht, lautet jedoch: „Auf das Chinesische, das keinerlei ,Morphologie" in unserem Sinne kennt und dessen Wortbildung rein syntaktisch verfährt, ist daher der Begriff des Morphems kaum, jedenfalls nur analogerweise zu übertragen" (Stetter 1999: 132, Anm. 29). Aus der reservatio mentalis gegenüber der analogen Verwendung eines in Bezug auf bestimmte Sprachsysteme definierten grammatischen Terminus auf Sprachen andersartigen Typus' wird so eine empirische "laienlinguistische Vorstellung“.

22 Um Missverständnisse zu vermeiden, habe ich hinzugefügt, in unserem, westlichen " Sinne", um damit das, was wir von Aristoteles bis Frege als formale Logik kennzeichnen, von anderen Verständnissen des Wortes „Logik“ abzugrenzen. 
Es ist keine Frage, dass in China (wie in Japan) Logiken entwickelt worden sind. Schlobinski referiert dazu einiges unter Bezug auf jüngere Darstellungen zu diesem Thema. Es ist auch keine Frage, dass die hier berichteten Überlegungen hochabstrakt und ,,sophisticated" zu nennen sind, dass sie mit Konzepten wie Negation, materialer Implikation, mit Variablen usw. arbeiten - einmal unterstellt, dass die referierten Übersetzungen der betreffenden chinesischen Begriffe korrekt sind. ${ }^{23}$ Aber das heißt nicht, dass die alten Chinesen deswegen ,formale' Logik betrieben hätten in dem Sinne, wie wir ihn erstmals explizit in Aristoteles' Analytica priora definiert und begründet haben ${ }^{24}$ mit einer Tradition, die bis zu Frege, Russell, Wittgenstein usw. reicht. Die bezüglich dieser Unterscheidung ,kritische" Frage lautet, ob denn in den Traditionen, auf die Schlobinski Bezug nimmt, auch ein Analogon zum Begriff der formalen Implikation entwickelt worden ist, d.h., aussagenlogisch gesprochen, zur Allgemeingültigkeit des Konditionals. ${ }^{25}$ Schon ein Blick in die ersten Abschnitte der Analytica priora macht klar, worum es in dieser Art von Logik geht: darum, allgemeingültig zu beweisen, dass aus einer gegebenen Prämisse etwas anderes folgt oder auch nicht. ${ }^{26}$ Und allgemeingültig heißt: ohne Rekurs auf die Bedeutung der in den betreffenden Argumenten verwendeten Begriffe. ${ }^{27}$ Man muss also semantisch fundierte Logiken von dem unterscheiden, was ,wir" in aristotelischer Tradition ,formale“ Logik nennen.

Was Schlobinski auszugsweise referiert, sind samt und sonders semantisch fundierte Argumente:

Dinge, die mehr gemeinsam haben, sind verschieden von Dingen, die weniger gemeinsam haben ...

$23 \mathrm{Vgl}$. Schlobinskis Referat S.132ff. Freilich bin ich mir nicht sicher, ob Herrn Schlobinski der Unterschied zwischen dem klar ist, was man in der formalen Logik ,materiale Implikation“ nennt, und dem, was man normalsprachlich „,material implizieren" nennt. Erstere beruht auf einer rein extensionalen Behandlung von Propositionen, und dies setzt ein entsprechendes logisches Semantik-Modell voraus, wie es von Frege, erstmals schon von Aristoteles entwickelt worden ist (vgl. Peri hermeneias Kap. V, 17a).

24 Hinzurechnen muss man als Grundlage der Analytiken die ,protologischen“ Schriften über die Kategorien und Peri hermeneias.

25 Das Konditional bzw. die Subjunktion in der üblichen Weise definiert als: $(p \rightarrow q) \leftrightarrow$ $\neg(\mathrm{p} \wedge\urcorner \mathrm{q})$.

26 Aristoteles kennt zwar noch nicht die moderne Fassung des Beweiskonzepts, die auf der aussagenlogischen Definition der formalen Implikation beruht, aber nicht nur der Idee nach, sondern auch in der Sache, in der Konzentration auf die Betrachtung von Satzformen, Quantoren und Negation ist das Konzept in den Analytica priora schon systematisch ausgearbeitet.

27 Dieser Begriff einer ,formalen“ Logik ist hier natürlich nicht in wenigen Sätzen erschöpfend darzulegen. Eine Einführung in die Problematik geben Tugendhat/Wolf in den ersten vier Kapiteln ihrer Logisch-semantischen Propädeutik (vgl. Tugendhat/Wolf 1983). 
Das Größte hat nichts außerhalb seiner selbst...

Ich gehe heute nach Yue, bin aber gestern dort angekommen ...

(vgl. Schlobinski 2001: $133 \mathrm{ff}$.).

Und die Frage, ob ein weißes Pferd ein Pferd ist oder nicht, ist, mit Frege betrachtet, eine Frage der Extension der Begriffe ,Pferd' und ,weißes Pferd', also wiederum eine semantische Frage, deren Klärung je vorausgesetzt ist, wenn man beginnt, formale Logik zu betreiben. Die Argumentationen, die hier andeutungsweise vorgeführt werden, wären quantorenlogisch überhaupt nicht darstellbar. ${ }^{28}$ Das letzte Paradoxon z. B. „Ich gehe heute nach Yue, bin aber gestern dort angekommen“, ${ }^{29}$ wird schon aussagenlogisch zu: „Ich gehe heute nach Yue und ich bin gestern in Yue angekommen." Was wäre daran noch paradox?

Dass es in der chinesischen Tradition hochentwickelte semantische Logiken gibt, ist unbestritten. ${ }^{30}$ Nur das war nicht mein Punkt. Mein Thema war in einem wesentlichen Punkt die Genese eines ,formalen" Denktypus, den wir in ,unserer" logischen und grammatischen Tradition haben. Und Stationen dieser Genese habe ich ja in den Platon und Aristoteles gewidmeten Kapiteln meines Buchs beschrieben.

Dass es - was die Grammatik betrifft - da ein philosophisches Problem gibt, welches auf die metaphysische Konzeption einer Grundform des Gedachten (des legomenon) zurückzuführen ist, habe ich formal in meiner Diskussion des chomskyschen Ansatzes gezeigt: Woher kommt das Anfangssymbol in einer Phrasenstrukturgrammatik, und was bedeutet es, wenn man die betreffende Leerstelle schlicht mit „Satz“ substituiert. Dies ist das Kardinalproblem einer Philosophie der Syntax, auch wenn der eine oder andere Linguist noch nie darüber nachgedacht hat (was er qua Linguist natürlich auch nicht unbedingt braucht). Wenn man aber ernstnimmt, was Chomsky als formale Neuerungen in die Linguistik eingeführt hat, und dazu gehört wesentlich das Prinzip der rekursiven Definition von sprachlichen Ausdrücken in einer formalen Metasprache, dann muss die Frage des Anfangssymbols für die theoretische Linguistik zu einem Problem mit empirischen Konsequenzen werden, und ein Blick in Saussures Cours genügt, um diese Annahme bestätigt zu finden. Die formalen, der aristotelischen Logik entstammenden Vorannahmen, die zur Formulierung eines, grammatischen' Satzbegriffs geführt haben, habe ich im

28 Schlobinski berührt in seiner Anmerkung 22 die Frage der Repräsentation von natürlichsprachlichen Argumenten durch eine formale Sprache (wobei ich seinem FregeZitat entnehme, dass ihm da eine quantorenlogische vorschwebt). Dieses Problem zu lösen lernt man, wenn man lernt, mit der Quantorenlogik zu operieren. Man vgl. als Einführung etwa Quine (1969: Kap.8).

29 Ich spare mir hier die quantorenlogische Formulierung wegen des technischen Aufwands, das ,,ich“ adäquat darzustellen.

30 Dies war auch Gegenstand des Gesprächs, von dem ich auf S. $12 \mathrm{f}$. meines Vorwortes kurz berichtet habe. 
Schlussabschnitt des Aristoteles-Kapitels von "Schrift und Sprache“ explizit aufgeführt und die Logik dieser Konstruktion beschrieben, sodass ich es hier bei diesem Hinweis belassen kann. Dass dieser Satzbegriff schriftinduziert ist und insofern hier ein kategoriales Problem für eine linguistische Universalientheorie liegt, hoffe ich zumindest ansatzweise gezeigt zu haben.

Dies war eine der Fragestellungen, denen ich in „Schrift und Sprache“ nachgegangen bin. Dass die formale Ausgestaltung von Logik und Grammatik mit der formalen Natur der Alphabetschrift zusammenhängt, ist sicher die Grundthese des Buchs. Ich bin aber nicht von ihr ausgegangen, sondern bin allmählich zu ihr gelangt, und ich habe sie im Vorwort auch entsprechend zu charakterisieren versucht: nämlich als eine „Überzeugung“ (S. 13), dass es diesen Zusammenhang gibt. Gleichwohl ist diese These nichts, das sich wissenschaftlich beweisen ließe. Dazu noch zwei abschließende Anmerkungen:

Zunächst noch einmal zurück zur vorgeblichen Relativismusthese mit ihrer zumindest bei Whorf - impliziten Determinismusannahme: Aus der und der Sprachstruktur folgt das und das Denken. Weil die Chinesen kein Alphabet hatten, konnten sie keine formale Logik entwickeln. Eine solche These ist als empirische These unhaltbar, denn sie ist weder verifizierbar noch falsifizierbar. Und darum ist sie philosophisch schlicht unsinnig. Humboldt hat solches auch nicht vertreten. Für ihn sind Sprachstrukturen ergon, der Einbildungskraft und dem Verstand gegebener Stoff, und nun gibt es nach Kant Köpfe und Pinsel. ${ }^{31}$ Die einen verfahren so mit dem, was ihnen dergestalt gegeben ist, die anderen so. Wo die Reise endet, ist nicht vorauszusagen.

Wohl aber kann eine gegebene Struktur eine Entwicklung in eine bestimmte Richtung begünstigen. Sprachen und Schriften sind Produkte evolutiver Prozesse. Und sie sind als solche selbst Momente der Evolution, etwa der der Stadt. Nun lese man in Leroi-Gourhans Le geste et la parole nach, mit welcher Meisterschaft es dieser Autor versteht, die Resultate seiner Wissenschaft zu einer Gesamtdeutung der Evolution des Menschen zusammenzufassen, ohne auch nur ein einziges Mal in ein deterministisches Denken zu verfallen.

An diesem Vorbild habe ich mich methodisch wie logisch zu orientieren versucht. Ich habe die Entwicklung der Alphabetschrift und die der formalen Logik bei Platon und Aristoteles vorausgesetzt und habe nach möglichen Zusammenhängen in dieser gegebenen Konstellation gefragt. Und da bin ich, mit Platons Hilfe, fündig geworden. Für Platon ist dieser Zusammenhang nämlich zwischen ,dialektischer' und ,grammatischer' episteme - ja offenbar gegenwärtig. Er spricht ihn wiederholt an, und den aus diesen Überlegungen gewonnenen formalen Umgang mit der Sprache demonstriert er einmal explizit: in der dialektischen Übung des Parmenides. Das habe ich, denke ich, hinreichend detailliert gezeigt. Natürlich hat nicht das Alphabet gedacht, sondern die

31 Kritik der Urteilskraft §47, B 183. 
„Vorsokratiker“", Sokrates, Platon usw. Und es hat vieles andere hinzukommen müssen, damit Platons Philosophie sich ausbilden konnte. ${ }^{32}$ Aber die Schrift hat ihre unübersehbaren Spuren in diesem Denken und in der Tradition hinterlassen, die es gestiftet hat, nicht nur als Thema - das ist bestens bekannt -, sondern ebenso subkutan, in der Ausprägung der Idee einer formalen Dialektik. Und da stand nun ein Schriftmedium zur Verfügung, das für die methodische Ausarbeitung einer solchen Idee bestens geeignet war, weil das Funktionsprinzip dieser Schrift darin bestand (und besteht), den Sinn des Gemeinten (to legomenon) indirekt dadurch kundzutun, dass man statt seiner seine Form darstellte, indem man sie ausbuchstabierte. Dass man in Platons Akademie über diesen Zusammenhang nachgedacht haben muss, belegt der vielzitierte erste Satz aus Aristoteles' Peri hermeneias. Dieser "Anfangssatz" ist ja eine Definition des Zusammenhangs von Denken und (schriftlicher) Sprache, die sich in den Diskussionen um eine Begründung der Logik in der Akademie abgespielt haben müssen. ${ }^{33}$ Diese Definition musste sich als zweckmäßig erwiesen haben, damit sie in diesem Text als Anfangsdefinition vorausgesetzt werden konnte. Und dass diese protologische Schrift mit diesem Satz beginnt, hat eben diesen Sinn: Wir können formale Logik betreiben, weil wir in den geschriebenen Wörtern ( $t a$ en tè graphè) die Form des Gedanken abgebildet haben. Zwar ist dies ,nur" ein Abbild, aber es erlaubt uns auf der anderen Seite den Aufbau einer in der Tat rein formal verfahrenden episteme. Den Konsequenzen, die sich daraus für eine Philosophie der Grammatik ergeben, bin ich in den letzten beiden, Humboldt und Wittgenstein gewidmeten Kapiteln von ,Schrift und Sprache“ nachgegangen. Dieser Problemzusammenhang war Gegenstand des Buchs, nicht ein dritter Aufguss der Sapir-Whorf-Hypothese. ${ }^{34}$

\section{Wer denkt eurozentristisch?}

Was nun - damit komme ich zum letzten Punkt - das Eurozentrismusproblem angeht, so ist zu fragen, wer denn eigentlich eurozentristisch denkt: Der, der konstatiert, dass die Evolution von Sprache und Schrift bei den Chinesen

32 Man vgl. hierzu Wolfgang Schadewaldts Darstellung der Entwicklung der griechischen Philosophie (Schadedewaldt 1978), wo viele der wesentlichen Vorbedingungen beschrieben sind.

33 Die Abfassung von Peri hermeneias fält sicher in die Zeit der Entstehung der Dialogserie Platons, die vom Kratylos bis zum Parmenides reicht: Vgl. Stetter (1999: 319 ff.).

34 Dies ist auch der Grund dafür, weshalb ich auf die reiche empirische Schriftforschung (die ich kenne und schätze) in diesem Buch nur am Rande eingegangen bin. Denn zu diesem Problem hat sie nichts geschrieben, auch Havelock nicht (den ich natürlich kannte). Hätte ich mich mit dieser empirischen Forschung auseinandergesetzt, so hätte ich methodisch wie materialiter ein anderes Buch schreiben müssen. 
offenbar anders, und zwar kategorial anders verlaufen ist als „,bei uns“, ${ }^{35}$ oder der, der zeigt, dass auch sie eine Logik hatten und diese bei Lichte besehen und bei wohlwollender Übersetzung in etwa genau so gut gemacht haben wie Aristoteles. Dies konstatiert selbstverständlich mit europäischen Kategorien. So wird man in die Irre geführt. Schlobinski ,,rekonstruiert“ den ,,Weißes-PferdDialog“ u. a. mithilfe der Unterscheidung von Extension und Intension. Nun ist aber nicht schwer zu sehen, dass sich der ganze Dialog nur deswegen so entfaltet, wie er sich entfaltet, weil die Unterscheidung dieser beiden Sinnebenen nicht vollzogen ist und offenbar (ich halte mich an den zitierten Ausschnitt) auch nicht vollzogen wird. ${ }^{36}$ Denn dann wäre der Dialog in drei Sätzen entschieden: Intensional betrachtet sind ,Pferd" und ,weißes Pferd" nicht dasselbe. Extensional betrachtet ist jedes weiße Pferd ein Pferd, aber nicht jedes Pferd ein weißes Pferd. Also kann die Fragestellung so nicht aufrechterhalten bleiben.

Wenn man nun annimmt, dass der Text logisch ernstzunehmen ist, kann man nicht gleichzeitig annehmen, dass der Autor, wäre ihm die Unterscheidung von Intension und Extension geläufig gewesen, das Problem nicht hätte lösen können. Also liegt der „Witz" des Dialogs vermutlich ganz woanders, in einer Problemstellung der kontextuellen chinesischen Philosophie, die uns bei der gegebenen Textlage verschlossen ist, jedenfalls eigens erschlossen werden müsste. Wie und warum Platon - um eine analoge Problemkonstellation zu nehmen - die handlungstheoretischen „Pendants“ von Extension und Intension, Referenz und Prädikation, gegen die physikalistische Semantik des Kratylos aufbietet und hierin so etwas wie eine ,Protologik' entwickelt, ${ }^{37}$ kann ja auch nur angemessen gewürdigt werden, wenn man den Kontext versteht, die Wahrheitsproblematik, die Sokrates zu Beginn des Dialogs in die (traditionelle) Debatte über das Problem der orthotes ton onomaton eingeführt hatte und die dann zentraler Gegenstand des Theaitetos ist. Solange man also die betreffenden Kontexte der zitierten Dialogfragmente nicht kennt, wäre m. E. eine methodische Haltung angebracht, die zunächst einmal davon ausgeht, dass die betreffenden Autoren etwas ganz anderes im Sinn hatten als das, was wir uns aus unserer Tradition über solche fragmentarischen Übersetzungen zusammenreimen.

In ähnlicher Richtung noch ein allerletztes Wort zu der von Schlobinski abschließend berührten Frage der Ökonomie des Alphabets: Warum sind die

35 Humboldt ist - bei aller Graecophilie - philosophisch, was das Chinesische angeht, doch diesen Weg gegangen: Es passt ihm zwar nicht in sein System, aber er erkennt seine Eigenständigkeit und seinen Rang dennoch an.

36 Dass dieser Dialog mit dem Nominalismus-Realismus-Problem der mittelalterlichen Philosophie nichts zu tun hat, folgt hieraus a forteriori. Denn diese Unterscheidung setzt die (entsprechende mittelalterliche) von denotatio (= Extension) und connotatio (= Intension) voraus. Sonst hätte sich das Problem ja gar nicht stellen können.

37 Kratylos 430a ff., vgl. dazu Stetter (1999: $322 \mathrm{ff}$.). Dort auch weitere Literaturverweise. 
Chinesen nicht längst von ihrem so schwer zu erlernenden Schriftsystem auf die Transkription in Pinyin umgestiegen? Es ,,stellt sich die Frage, warum in China eine Schrift gebraucht wird, bei der ca. 3000 Zeichen notwendig sind, um eine Zeitung zu lesen, und nicht eine höchst ökonomische Alphabetschrift mit einem Zeicheninventar von 27 Graphemen. " ${ }^{38}$ Wiederum ein Kategorienfehler: Zwar wird hier zweimal das Wort „Zeichen“ verwendet, um die Korrelate des Vergleichs $\mathrm{zu}$ bezeichnen, doch dieses Wort bedeutet in den beiden Verwendungsfällen Verschiedenes:

Wird von einem Han ze oder Kanji als von einem Zeichen gesprochen, so versteht man darunter - in linguistischer Dogmatik - eine Einheit, an der die beiden Zeichenaspekte von signifiant und signifié in der Form auszumachen sind, dass man sagen kann: Die Zeichengestalt XYZ hat (in dieser Verwendung) die und die Bedeutung. Dieses Kanji (dabei zeigt man etwa auf ein Zeichen in einer Speisekarte eines japanischen Restaurants) bedeutet „,Tintenfisch“. Buchstaben haben aber nichts, das in irgendeiner Weise als ihre Bedeutung (im linguistischen oder logischen Sinn) anzusprechen wäre. Sie werden verwendet, um Wörter zu schreiben. Jedes Wort (in einer Alphabetschrift) ist von jedem anderen Wort derselben Schriftsprache durch eine besondere Buchstabenfolge unterschieden. ${ }^{39}$ Erst diese Wörter sind in linguistischem Sinne Zeichen. ${ }^{40}$ Die Buchstaben sind systematisch den Phonemen vergleichbar, und auch die sind für sich betrachtet keine Zeichen, sondern haben lediglich ,,bedeutungsdifferenzierende" Funktion.

Also muss man, wenn schon, den Kanji bzw. Han ze die geschriebenen Wörter unserer Sprache vergleichen, und da nimmt sich die Frage der Ökonomie nun ganz anders aus: Auch wir speichern - wie die Chinesen oder Japaner - ganze Wortbilder, nur sind die des Alphabets abstrakter, weil a) über orthographische Normen und b) über Regelungen der Buchstabengestaltung geregelt, also nicht sichtbar wie die Strichkombinationen in den chinesischen Zeichen. ${ }^{41}$ Wieso und in welcher Hinsicht ist ein kleines Zeichenrepertoire „ökonomischer" als ein großes? Doch nicht per se: Das Zeichenrepertoire „, 0,1 “ ist ohne Zweifel kleiner

38 Schlobinski (2001: 144). Ähnliche Fragen - man könnte auch sagen Vorurteile habe ich gelegentlich in Briefen von in Japan lebenden native speakers des Englischen bzw. Amerikanischen an die Japan Times gefunden.

39 Die wenigen Fälle der Homonyme wie „Ball“ oder „Kiefer“ hier vernachlässigt.

40 Sprachsystematisch könnte man hier noch die Ebene der Morpheme berücksichtigen. Doch das ist für den faktischen Gebrauch der Alphabetschrift irrelevant. Ihre kleinsten, durch die beiden orthographischen Register der Buchstabenfolge und der Getrennt- und Zusammenschreibung konstituierten Zeicheneinheiten sind die durch Spatien u. ä. Mittel voneinander getrennten Wörter.

41 Dass $d$ und $b, p$ und $q, u$ und $n$ verschiedene Zeichengestalten sind, ergibt sich erst aus einer Regel, welche z. B. besagt, dass ein um seine Längsachse gedrehtes Zeichen ein anderes Zeichen ist, eine Regelung, die für Großbuchstaben nicht gilt. Diese Regel kann man nicht sehen wie eine Strichekombination, man muss sie verstehen. 
als das Repertoire ,0,1,2,3,4,5,6,7,8,9“. Also wollen wir doch unser Dezimalsystem durch das Dualsystem ersetzen: $0,01,10,11$ usw. Dass nun $4 \approx 100$ und $8 \approx 1000$, mag noch hingehen, aber wie war das mit 47 ? Die Zeichenkombinationen des Dualsystems sind schwieriger zu memorieren, weil ihre Bedeutung über Regeln vermittelt ist, während das Dezimalsystem einen größeren Fundus an Zeichen hat, die Bedeutungsunterschiede durch ihre Form direkt anzeigen. Wie große Schwierigkeiten Anfängern Zehner- oder Hunderterüberschreitungen machen, ist bekannt. Und trotz der notorischen Schwierigkeiten, die unsere Kinder mit der Orthographie haben ${ }^{42}$ - ein ,interner ${ }^{6}$ Teil der Alphabetschrift, ohne den das System nicht zu gebrauchen wäre - und trotz der bis heute weithin unbekannten Schwierigkeiten in der Identifizierung so ähnlicher Gestalten wie b, d, p, q; m, n, u, v; c, e, o usw., die sicher auseinandergehalten werden können erst dann, wenn man für jede der ähnlichen Gestalten eine hinreichende Anzahl verschiedener Verwendungskontexte gelernt hat, und jeder dieser Kontexte besteht dann wiederum aus ebenso abstrakten Buchstaben, die mit anderen verwechselt werden können (weil es eben nur so wenige gibt) ...- trotz all dieser eigentlich vor Augen liegender Dinge hält sich hartnäckig der Mythos, die Alphabetschrift sei das im Vergleich mit dem Chinesischen oder Japanischen leichter zu lernende Schriftsystem.

Es könnte durchaus anders sein. Deshalb wäre mein Plädoyer, zunächst einmal zu versuchen, die verschiedenen Schriftsysteme in ihrer Verschiedenheit zu verstehen und so auch das Alphabet - bei all seiner Universalität - doch als eine besondere Problemlösung in der Evolution der Schrift zu begreifen. ${ }^{43}$ Den in der Alphabetschrift Literalisierten kommt deren indirekte, höchst abstrakte Weise, den Gedanken mit graphischen Mitteln darzustellen, als das Natürlichste und Leichteste der Welt vor. Was die chinesische Schrift angeht, so könnten sie an ihr lernen, wie ein Graphismus ohne den Umweg über die Lautsprache es leisten kann, Gedanken mit einer ähnlichen logischen Präzision und einer vergleichbaren semantischen Differenzierungstiefe darzustellen wie im Medium des Oralen. Dahin sind ,wir“ - allerdings mit jeweils beschränkten Semantiken - bislang nur im Bereich symbolischer Sprachen gelangt, wo es denen, die sie beherrschen, selbstverständlich ist, dass man in ihren Formen denken kann, ohne die in ihnen artikulierten Gedanken jeweils erst ins Natürlichsprachliche übersetzen zu müssen.

42 Absolventen des 10. Schuljahrs beherrschen sie bei weitem noch nicht.

43 Die Besonderheiten dieser Problemlösung und die spezifischen Schwierigkeiten, die daraus für das Erlernen der Alphabetschrift resultieren, habe ich in einer Arbeit beschrieben, die im Zusammenhang eines von der Fritz-Thyssen-Stiftung geförderten Forschungsprojekts entstanden ist. Vgl. Stetter 2001. 


\section{Literatur}

Humboldt, Wilhelm von (1906): Lettre à Monsieur Abel-Rémusat, GS V, 254-308.

Humboldt, Wilhelm von (1969): Ueber die Buchstabenschrift und ihren Zusammenhang mit dem Sprachbau. In: Flitner, Andreas/Giel, Klaus (Hgg.): Werke in fünf Bänden, Bd. III, Schriften zur Sprachphilosophie. 3., durchges. Auflage Darmstadt: Wissenschaftliche Buchgesellschaft, 82-112.

Müller-Yokota, Wolfram (1994): Die chinesische Schrift. In: Günther, Hartmut/Ludwig, Otto (Hgg.): Schrift und Schriftlichkeit. Ein Interdisziplinäres Handbuch internationaler Forschung. Berlin/New York: de Gruyter. 1. Halbband, 347-382.

Quine, Willard v.O. (1969): Grundzüge der Logik. Aus dem Englischen übers. von Dirk Siefkes. Frankfurt a. M.: Suhrkamp (= stw 65).

Schadewaldt, Wolfgang (1978): Die Anfänge der Philosophie bei den Griechen. Die Vorsokratiker und ihre Voraussetzungen. Tübinger Vorlesungen Band 1. Frankfurt a. M.: Suhrkamp. (= stw 218)

Schlobinski, Peter (2001): Zum Prinzip des Relativismus von Schriftsystemen - die chinesische Schrift und ihre Mythen. In: Zeitschrift für Sprachwissenschaft 20, 117-146.

Stetter, Christian (1999): Schrift und Sprache. Frankfurt a. M.: Suhrkamp (= stw 1415).

Stetter, Christian (2001): Über die Schwierigkeiten, die Alphabetschrift zu erlernen. (Im Druck)

Tugendhat, Ernst/Wolf, Ulrike (1983): Logisch-semantische Propädeutik. Stuttgart: Reclam.

Eingegangen: 27.9.2001

Christian Stetter, Germanistisches Institut der RWTH Aachen, Eilfschornsteinstraße 15, 52062 Aachen 